\title{
Asymptomatic Lacrimal Flow Abnormalities in Patients with Septal Deviations and Turbinate Hypertrophy
}

\author{
V. Cervelli · G. Gravante - G. M. Colicchia - M. Grimaldi · D. J. Bottini · \\ P. L. Torcia - F. Garzione
}

Received: 27 April 2007 / Accepted: 22 May 2007/Published online: 8 August 2007

(C) Springer Science+Business Media, LLC 2007

\begin{abstract}
Background: This study aimed to investigate the lacrimal flow in patients affected by septal deviations and turbinate hypertrophy and to evaluate changes after rhinoseptoplasty with dacryocystography (DCT) and computed tomographic dacryocystography (CT-DCT).

Methods: The study prospectively recruited patients having septal deviations with or without turbinate hypertrophy who underwent surgical evaluation for correction of their respiratory symptoms and were not referred for epiphora. Patients were excluded if they had undergone surgery for cranial vault defects or had experienced septal deviations after traumatic accidents. All patients were studied with DCT and CT-DCT preoperatively and postoperatively.

Results: A total of 24 patients (10 men and 14 women) were recruited for the study. Of these patients, $11(45.8 \%)$ had a reduced flow of the medium contrast due to a partial obstruction at the level of the internal ostium. All 11 patients had septal deviations and turbinate hypertrophy, whereas 8 patients had a unilateral obstruction (72.7\%), and 3 patients had a bilateral obstruction (27.3\%). All flows were corrected after surgery.

Conclusions: The safe and well-tolerated radiologic techniques performed in this study provided detailed
\end{abstract}

V. Cervelli · G. M. Colicchia · M. Grimaldi · D. J. Bottini Department of Plastic Surgery, University of Tor Vergata in Rome, Rome, Italy

G. Gravante $(\square)$

Department of General Surgery, University of Tor Vergata in Rome, via U. Maddalena 40/a, 00043 Ciampino, Rome, Italy e-mail: ggravante@hotmail.com

P. L. Torcia - F. Garzione

Hospital S. Carlo of Nancy, Rome, Italy imaging of the lacrimal outflow system. A high incidence of partial obstruction to the internal ostium was found in patients with septal deviations, turbinate hypertrophy, and no lacrimal symptoms, suggesting a frequent presymptomatic condition.

Keywords Computed tomographic dacryocystography . Dacryocystography · Lacrimal ducts · Rhinoseptoplasty . Septal deviation · Turbinate hypertrophy

The anatomic proximity of the lacrimal system to the nose renders possible its involvement during rhinoseptoplasty and can result in postoperative transient or permanent obstructions to the lacrimal flow [1]. In contrast, correction of septal deviations and reduction of turbinate hypertrophy usually reduce the lacrimal engorgement at the level of the internal inferior nasal meatus, ordinarily resulting in an amelioration of the epiphora.

Dacryocystography (DCT) and computed tomographic DCT (CT-DCT) are important radiologic tools for evaluating the lacrimal duct anatomy and pathology [2-4]. These techniques have been used to study the effect of lateral osteotomies, external and endoscopic paranasal sinus surgery, and dacryocystorhinostomy on the lacrimal apparatus [5-9]. In the current study, we used these techniques to evaluate the incidence of outflow alterations among patients who had septal deviations with or without turbinate hypertrophy but asymptomatic for epiphora and postoperative changes occurring after corrective rhinoseptoplasty.

\section{Materials and Methods}

We prospectively recruited patients having septal deviations with or without turbinate hypertrophy who underwent 
Table 1 Septal deviations versus deviations with turbinate hypertrophy: Descriptive statistics and comparison among groups

\begin{tabular}{llcl}
\hline & Septal deviations $(\mathrm{n}=4)$ & $\begin{array}{l}\text { Septal deviations with } \\
\text { turbinate hypertrophy } \\
(\mathrm{n}=20)\end{array}$ \\
\hline Age (years): n (range) & $34(22-47)$ & $32(20-44)$ & $\mathrm{NS}$ \\
Sex (males): $\mathrm{n}(\%)$ & $2(50)$ & $8(40)$ & $\mathrm{NS}$ \\
Overall obstructions: $\mathrm{n}(\%)$ & 0 & $11(55)$ & 0.04 \\
Unilateral obstructions: $\mathrm{n}(\%)$ & 0 & $8(40)$ & $\mathrm{NS}$ \\
Bilateral obstructions: $\mathrm{n}(\%)$ & 0 & $3(15)$ & $\mathrm{NS}$ \\
\hline
\end{tabular}

NS, not significant

surgical evaluation for correction of their respiratory symptoms and were not referred for any lacrimal symptoms. We excluded those who underwent surgery for cranial vault defects or experienced septal deviations after traumatic accidents. All patients were evaluated using DCT and CT-DCT at the S. Carlo Hospital of Nancy and underwent surgery at the Plastic and Reconstructive Surgery Department of the University of Rome "Tor Vergata."

The patients received a complete preoperative study with extensive photographic documentation, washing of the lacrimal ducts and subsequent probe exploration, anterior and posterior rhinoscopy, DCT, and CT-DCT. All DCTs and CT-DCTs were performed using a hydrosoluble contrast medium injected with a constant and continuous pressure into the lacrimal ducts. Anesthesia was induced with orotracheal intubation and controlled hypotension to reduce intraoperative bleeding.

The operation adopted was a conservative rhinoseptoplasty with lussation of turbinates. Follow-up evaluation consisted of weekly controls during the first month, then every 3 months for the first 6 months, and finally a second radiologic DCT and CT-DCT at postoperative month 6 .

Our goals were to study preoperative lacrimal flow in patients affected by septal deviations and turbinate hypertrophy and to evaluate changes after rhinoseptoplasty.

\section{Statistical Analysis}

All data analyses and calculations of sample size were performed using the Statistical Package for the Social Sciences Windows version 13.0 (SPSS, Chicago, IL, USA). Descriptive statistics for quantitative continuous variables were the median and range (minimum and maximum) for nonparametric variables after exclusion of normality with histograms, as well as Kolmogorov/Smirnov and Shapiro Wilk testing. Descriptive statistics for qualitative categorical variables were presented with frequencies.

Comparison for group homogeneity was performed using Student's $t$-test for continuous variables and the
Fisher's test for categorical variables. All $p$ values less than 0.05 were considered significant.

\section{Results}

From September 2006 to September 2007, we recruited 24 patients (10 men and 14 women) for the study. The mean age of patients was 33 years (range, 20-47 years). All patients were affected by septal deviations, and 20 patients also had turbinate hypertrophy $(83.3 \%)$. Eleven patients (45.8\%) had reduced flow of the medium contrast due to a partial obstruction at the level of the internal ostium, as evidenced by the DCT and CT-DCT (Figs.1-3). All of them $(55 \%)$ were in the group of 20 patients with septal deviations and turbinate hypertrophy, and such a difference was significant $(p<0.05$; Table 1$)$. Eight patients had a unilateral obstruction $(72.7 \%)$, and three had a bilateral obstruction $(27.3 \%)$.

Every preoperative septal deviation and turbinate hypertrophy was corrected, and a resolution of respiratory symptoms was observed in all patients. The DCT and CTDCT scans verified the correction, corresponding to functional amelioration of the respiratory function and the normal flow of the contrast medium throughout the lacrimal ducts into the rhinopharinx (Figs.1-3). Neither damage to the lacrimal ducts nor de novo insurgence of epiphora was found after the operation.

\section{Discussion}

Every day, an increasing number of patients ask for aesthetic procedures to ameliorate their social or psychological conditions. However, during rhinoplasty, it is possible that lacrimal ducts are damaged due to the strict relationship of these structures with the nose, resulting in alterations of the normal lacrimal flow [1]. Obstructions can be transient, more frequent, or permanent [1]. Transient obstructions derive from the mucosal edema created with the surgical trauma, whereas permanent obstructions 
Fig. 1 Right lacrimal duct obstruction (red) in a 37-yearold man affected by septal deviation. Upper panel: Preoperative computed tomography $(\mathrm{CT})$ scan of the axial section (left) and magnification (right). Middle panel: Preoperative CT scan of the coronal section (left) and magnification (right). Lower panel: Preoperative CT dacryocystography (CT-DCT) of the sagittal section (left) and postoperative resolution after rhinoseptoplasty (right)
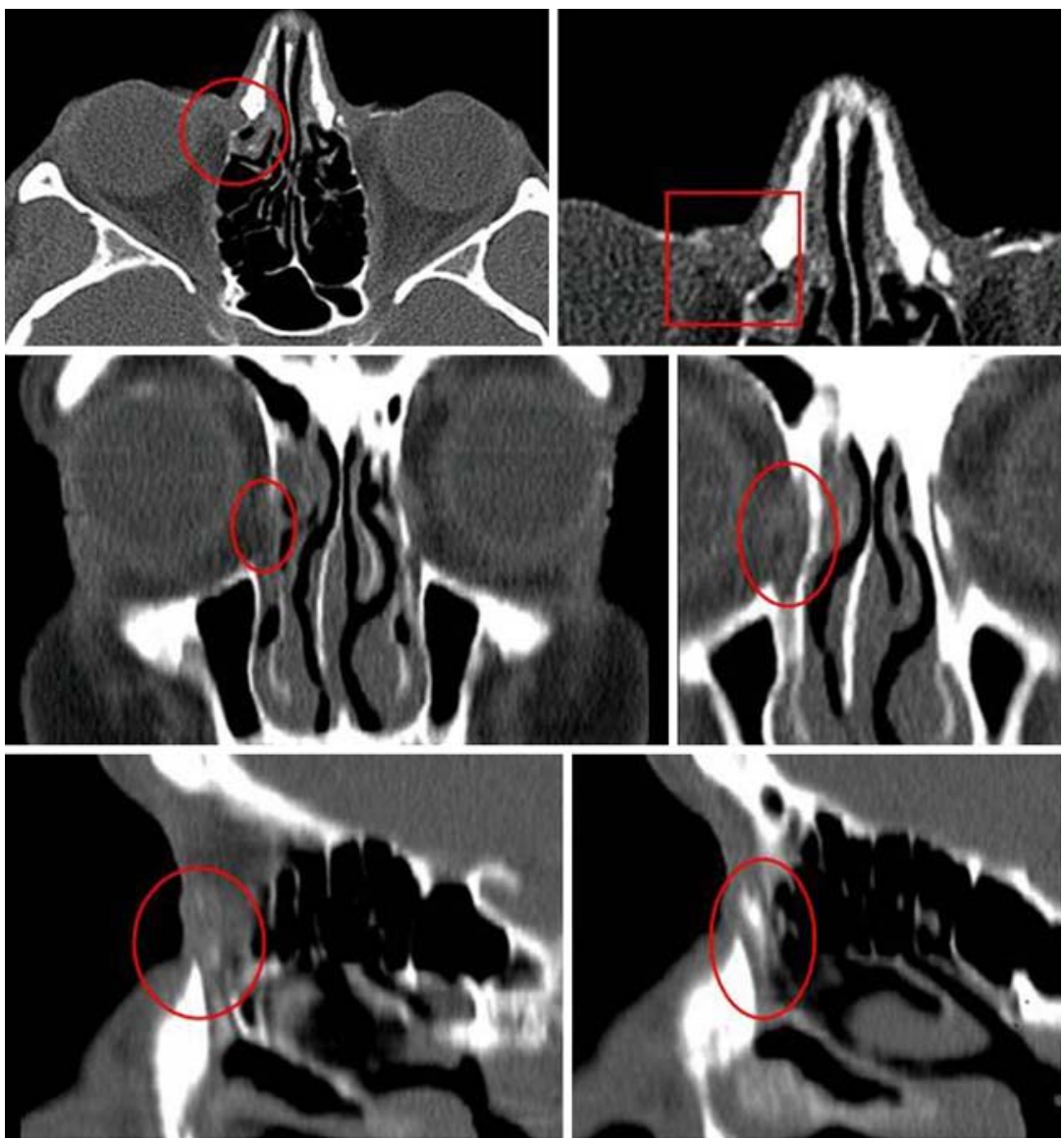

derive from damage to the lacrimal sac or nasolacrimal openings caused by surgical dislocations of nasal bones [1].
Although rhinoseptoplasty can adversely affect the lacrimal flow, it also can correct its alterations. In fact, septal deviations and turbinate hypertrophy are frequent causes of
Fig. 2 Right lacrimal duct obstruction $(r e d)$ in a 25-yearold woman affected by septal deviation and turbinate hypertrophy. Upper panel: Preoperative computed tomography (CT) scan of the axial section (left) and magnification (right). Middle panel: Preoperative CT scan of the coronal section (left) and magnification (right). Lower panel: Preoperative

dacryocystography (DCT) of the sagittal section (left) and postoperative resolution after rhinoseptoplasty and partial turbinectomy (right)
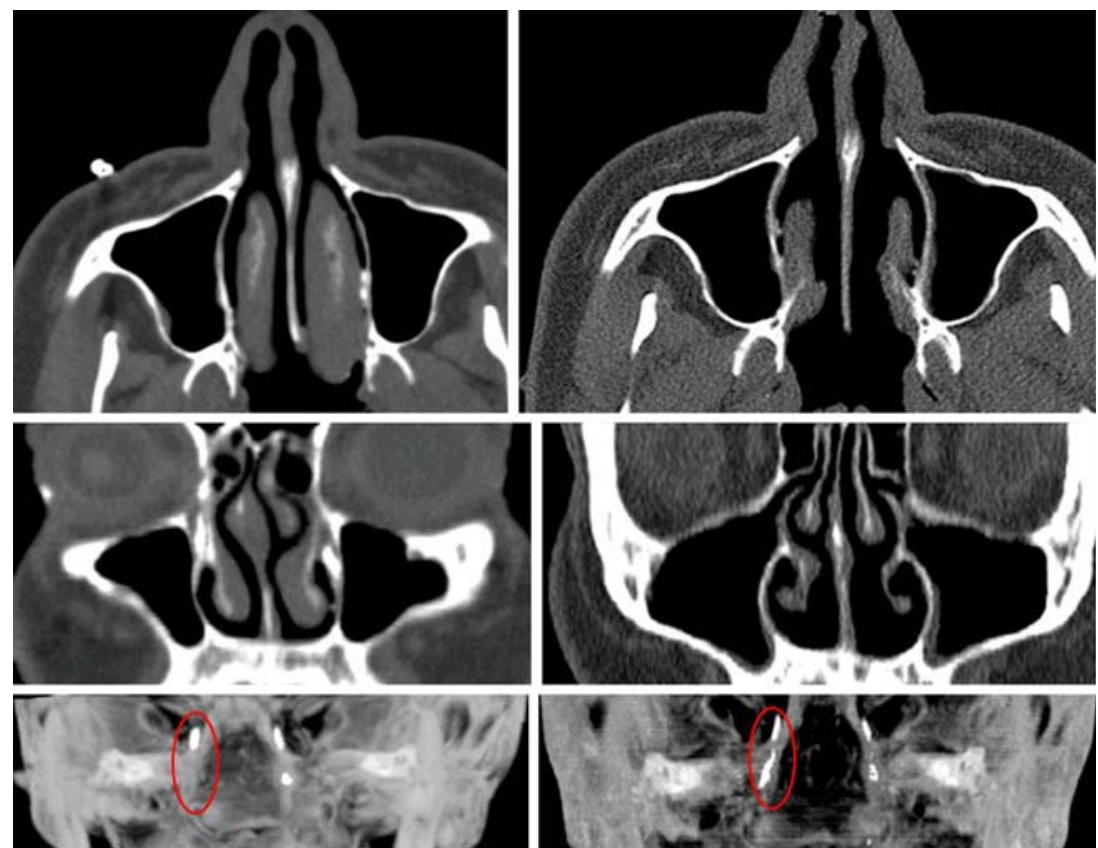
Fig. 3 Bilateral lacrimal duct obstruction in a 46-year-old woman with septal deviation. Upper panel: Preoperative (left) and postoperative (right) computed tomography (CT) scan of the axial section. Middle panel: Preoperative (left) and postoperative (right) CT scan of the coronal section. Lower panel: Preoperative (left) and postoperative (right) CT-DCT sagittal section
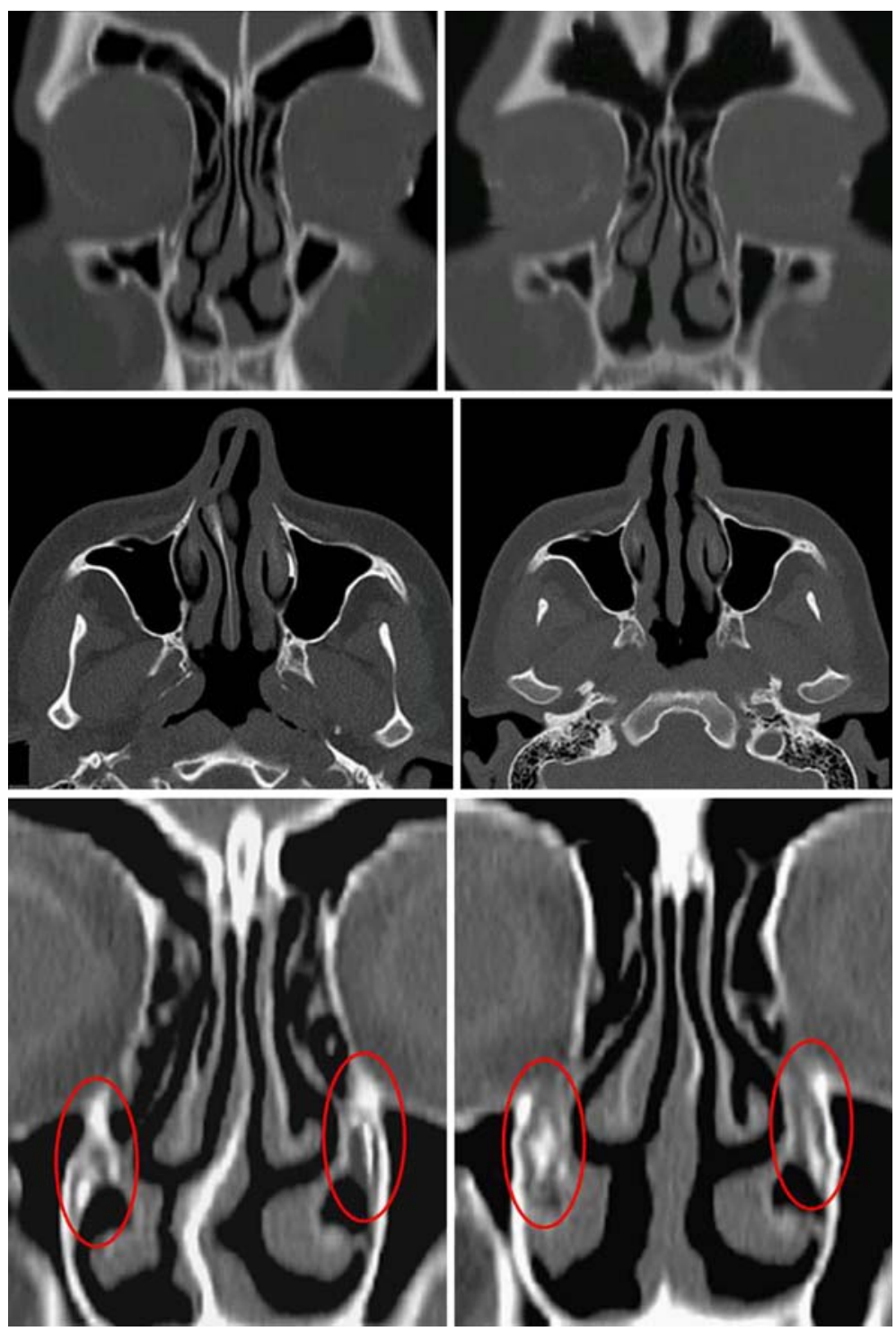

reduced lacrimal outflow from the inferior nasal meatus, and the surgical correction of both factors usually resolves the problem in these patients [10-12]. In the study of Gray [10], the presence of septal deformity and the close correlation with the affected side has been found in every patient with continued epiphora requiring probing and syringing of the nasolacrimal ducts and manipulation of the palate and septum, often with medial dislocation of the anterior end of the inferior turbinates, or a simple turbinate fracture with a hemostat, all applied as helpful techniques for treatment $[10,12]$. In the current study, we focused on patients affected by septal deviations and turbinate hypertrophy without lacrimal symptoms to study the incidence of subclinical anomalies of the lacrimal flow and to evaluate eventual corrections obtained with surgery.

Different radiologic techniques are used to diagnose and evaluate lacrimal duct alterations. Usually, DCT is selected to define the lumen of the lacrimal drainage system in dacryocystitis, dacryoceles, dacryoliths, and developmental abnormalities. It also provides supplemental information concerning benign and malignant tumors, principally to differentiate an intrinsic lesion from an extrinsic lesion. Both CT-DCT and magnetic resonance (MR)-DCT reproducibly and noninvasively show the lacrimal drainage apparatus and allow a better physiologic examination than cannulation DCT. The use of CT-DCT is indicated for delineation of palpable mass lesions such as cysts, benign and malignant tumors, and diseases, especially in the paranasal sinuses, affecting the lacrimal apparatus secondarily. Clinicians can perform MR-DCT without administering ionizing radiation or contrast material. It is useful for differentiating fluid from solid masses and for defining tumor extension from the sac into the duct as well as anatomic regions outside the sac and duct [13-14]. 
However, MR-DCT cannot show adjacent bone anatomy and has shown the smaller drainage structures less consistently than CT-DCT [15]. For this reason, we decided to use DCT and CT-DCT for our patients.

In our study, DCT and CT-DCT were useful tools for visualizing the anatomy of the lacrimal structures and their relationship with nearby bones. We found that $45.8 \%$ of patients with septal deviations with or without turbinate hypertrophy had asymptomatic partial obstructions of the lacrimal flow that derived from partial closure of the internal ostium by the turbinates. The ease of execution, the high quality of the images with the possibility of magnification, and the dynamic evaluation all were characteristics that contributed to discriminating soft tissues from bones and to diagnosing the cause of the obstruction precisely. The postoperative follow-up evaluation showed that correction of the septum and partial turbinectomy completely resolved the altered lacrimal flow in all patients, as evidenced by DCT and CT-DCT that showed normal outflows of the medium contrast.

Finally, we believe that turbinate hypertrophy was the most important factor in determining obstructions because all cases involved septal deviations with turbinate hypertrophy. No cases involved septal deviations alone, and the difference between the groups was significant. However, bilateral partial cases could be explained by the concomitant presence of septal deviation with another contributing factor such as. chronic mucosal inflammation. This hypothesis needs further investigation in future studies.

\section{Conclusions}

Two important radiologic tools, DCT and CT-DCT, allow the plastic surgeon to precisely find and estimate asymptomatic alterations of the normal lacrimal outflow in patients with septal deviations and turbinate hypertrophy. We found a high incidence of partial closures of the internal ostium (45.8\% of asymptomatic patients). The lacrimal duct anatomy was precisely assessed, suggesting that both septal deviation and turbinate hypertrophy were the most probable causes for the outflow reduction. This also was confirmed postoperatively when the radiologic evaluation found a normal outflow after correction.

\section{References}

1. Osguthorpe JD, Calcaterra TC (1979) Nasolacrimal obstruction after maxillary sinus and rhinoplastic surgery. Arch Otolaryngol 105:264-266

2. Ansari SA, Pak J, Shields M (2005) Pathology and imaging of the lacrimal drainage system. Neuroimaging Clin North Am 15:221237

3. Weber AL, Rodriguez-DeVelasquez A, Lucarelli MJ, et al. (1996) Normal anatomy and lesions of the lacrimal sac and duct: Evaluated by dacryocystography, computed tomography, and MR imaging. Neuroimaging Clin North Am 6:199-217

4. Freitag SK, Woog JJ, Kousoubris PD, et al. (2002) Helical computed tomographic dacryocystography with three-dimensional reconstruction: A new view of the lacrimal drainage system. Ophthal Plast Reconstr Surg 18:121-132

5. Yigit O, Cinar U, Coskun BU, et al. (2004) The evaluation of the effects of lateral osteotomies on the lacrimal drainage system after rhinoplasty using active transport dacryocystography. Rhinology 42:19-22

6. Massoud TF, Whittet HB, Anslow P (1993) CT-dacryocystography for nasolacrimal duct obstruction following paranasal sinus surgery. Br J Radiol 66:223-227

7. Unlu HH, Goktan C, Aslan A, et al. (2001) Injury to the lacrimal apparatus after endoscopic sinus surgery: Surgical implications from active transport dacryocystography. Otolaryngol Head Neck Surg 124:308-312

8. Amin M, Moseley IF, Rose GE (2000) The value of intubation dacryocystography after dacryocystorhinostomy. $\mathrm{Br} \mathrm{J}$ Radiol 73:604-607

9. Gokcek A, Argin MA, Altintas AK (2005) Comparison of failed and successful dacryocystorhinostomy by using computed tomographic dacryocystography findings. Eur J Ophthalmol 15:523-529

10. Gray LP (1980) Relationship of septal deformity to snuffly noses, poor feeding, sticky eyes, and blocked nasolacrimal ducts. Int J Pediatr Otorhinolaryngol 2:201-215

11. Wesley RE, Bond JB (1986) Intranasal procedures for successful lacrimal surgery. Ophthal Plast Reconstr Surg 2:153-157

12. Wesley RE (1985) Inferior turbinate fracture in the treatment of congenital nasolacrimal duct obstruction and congenital nasolacrimal duct anomaly. Ophthalmic Surg 16:368-371

13. Weber AL, Rodriguez-DeVelasquez A, Lucarelli MJ, et al. (1996) Normal anatomy and lesions of the lacrimal sac and duct: Evaluated by dacryocystography, computed tomography, and MR imaging. Neuroimaging Clin North Am 6:199-217

14. Manfre L, de Maria M, Todaro E, et al. (2000) MR dacryocystography: Comparison with dacryocystography and CT dacryocystography. AJNR Am J Neuroradiol 21:1145-1150

15. Caldemeyer KS, Stockberger SM Jr, Broderick LS (1998) Topical contrast-enhanced CT and MR dacryocystography: Imaging the lacrimal drainage apparatus of healthy volunteers. AJR Am J Roentgenol 171:1501-1504 